\title{
EDITORIAL
}

\section{COVID-19 STILL ON CENTER STAGE IN ONCOLOGY DEBATES}

\author{
A. Giordano ${ }^{1,2}$, C. Pinto ${ }^{3}$, F. Pentimalli 4 \\ 1 Department of Medical Biotechnologies, University of Siena, Siena, Italy \\ 2 Sbarro Institute for Cancer Research and Molecular Medicine, Center for Biotechnology, College of Science and \\ Technology, Temple University, Philadelphia, PA, USA \\ ${ }^{3}$ Oncologia Medica, Clinical Cancer Center, AUSL-IRCCS, Reggio Emilia, Italy \\ ${ }^{4}$ Cell Biology and Biotherapy Unit, Istituto Nazionale Tumori-IRCCS-Fondazione G. Pascale, Naples, Italy
}

\section{CORRESPONDING AUTHOR:}

Francesca Pentimalli

Cell Biology and Biotherapy Unit

Istituto Nazionale Tumori

IRCCS-Fondazione G. Pascale

via Mariano Semmola 52

I-80131 Naples, Italy

E-mail: f.pentimalli@istitutotumori.na.it

Doi: $10.48286 /$ aro.2021.11

The Covid-19 pandemic, which is still far from over, is continuing to take center stage in most debates on current cancer research and care. A year after Covid-19 outbreak and the first lockdown, it is clear that the disease had a heavy toll on cancer patients impacting on every stage from screening to diagnosis and treatment (1-3). With the availability of antiSARS-CoV2 vaccines, recent debates focused also on the vaccination strategies for cancer patients and for those eligible to access oncology clinical trials $(4,5)$. In our previous issue we discussed how the pandemic affected the number of new cancer diagnoses, showing a reduction compared to the same period in the preceding year $(1,6)$, and our authors presented some of the challenges faced from oncologists during the first wave of the pandemic $(7,8)$. In this issue of Annals of Research in Oncology, Lucia Fratino and Diego Serraino focus on some clear cut questions regarding SARS-CoV-2 infection, cancer, cancer therapies and immune suppression which demand attention and, possibly, guidelines, although recommendations for the management of cancer patients in the context of SARS-CoV-2 infection have been mostly produced outside the traditional "Evidence Based" benchmark because of the urgency (9).
Luigi Cavanna and colleagues, who pioneered home treatment of Covid-19 patients, report in this issue on their successful experience in a Northern Italy area, which was heavily affected by the disease during the first wave (10). Cavanna and colleagues, defined as 'heroes of the front lines' by the Time (11), show how the early diagnosis and treatment of the infection in cancer patients could avoid hospitalization and death in their cohort, strongly suggesting that early home management and monitoring through telemedicine should be implemented (10). Daniela Fanni and co-authors present a case of a 63-year-old patient affected by Covid-19 with progressive respiratory failure. Histological evidence allowed the diagnosis of pulmonary capillary hemangiomatosis $(\mathrm{PCH})$, which reinforces the hypothesis that the endothelial dysfunction, capillary thrombosis and neoangiogenesis induced by SARS-CoV-2 infection could evolve toward $\mathrm{PCH}$, leading to the disruption of lung architecture (12). For the launch of Annals of Research in Oncology the chief editors, Antonio Giordano and Carmine Pinto, with the Edra Chief business and content officer Ludovico Baldessin, in collaboration with the Center for American Studies (https://centrostudiamericani. 
org/), held an online conference on The value of scientific knowledge pre \& post Covid. (https://www.youtube.com/watch?v=7ed-LSIN9Lk). The conference was introduced by Roberto Sgalla, Director of the Center for American Studies, which is engaged in promoting knowledge and research activity, building bridges across disciplines and entities. In particular, Beatrice Lorenzin, former Italian Minister of Health, participated as coordinator of the Health \& Science Bridge Programme. Ludovico Baldessin interviewed the speakers on how to improve scientific communication and how to advance medical and scientific progress following the Covid-19 experience. Lorenzin stressed the importance of the education of young generations to promote a scientific culture and avoid misunderstanding or fake news dissemination. Giordano discussed the US system in which patients are more involved in decision making processes compared to other countries; for example, they participate in the peer review process established to assign governmental funding. Pinto discussed how in Italy oncology research performs well, as judged by the number of publications produced, despite having much less public funding compared to other countries. Pinto also highlighted the importance of having a unified health system that is too often fragmented across various regions. For example, the presence of a unique Ethical Committee during the Covid-19 pandemic allowed the evaluation of about 144 studies in a 3-month timeframe, which led to the rapid approval of $32 \%$ of the proposed studies; overall, an incredible acceleration compared to the usual bureaucracy-driven pathways, which slow down timely and competitive scientific research. Then, Giovanni Baglio from the Italian National Agency for Regional Health Services (Agenas) discussed how the pandemic affected the usual evidence-based-driven decision-making process required to take health decisions by regulatory agencies changing the perspective towards an 'evidence-making interventions'. Such new perspective, which gives values to the context and takes into account the available resources, promises to become a new useful standard of practice for the elaboration of health recommendations. Besides Covid-19 impact on cancer, the second issue of Annals of Research in Oncology brings together a set of articles encompassing different areas of cancer research and treatment.

Perrone and coauthors report the results of an observational retrospective study performed on a cohort of 270 patients of epithelial ovarian cancer. Unfortunately, this cancer type often relapses requiring sec- ondary cytoreduction surgery, so the authors investigated whether the primary treatment could affect the pattern of recurrent disease and secondary surgery. Their study suggests that the primary treatment, consisting either of primary debulking surgery or neoadjuvant chemotherapy followed by interval debulking surgery, should be considered among the selection variables for secondary cytoreduction surgery further implementing other parameters such as clinical score based on ascites, performance status, and absence of residual disease upon primary surgery (13).

Lucia Mangone and colleagues present in this issue data from the Italian Association of Cancer Registries (AIRTUM Working Group) on the epidemiology of neuroendocrine neoplasms (NEN) in Italy. The authors collected data from thirty-eight cancer registries spanning the 1976-2012 period concerning a total of 9,707 NENs. The study showed that the incidence of NENs increased almost sevenfold since 1976 and a fifth of all cases showed an association with another cancer, which might affect clinical management decision strategies (14).

Alessandro Lambiase and colleagues review the latest advancements in the classification and treatment of ocular surface tumors, which include a range of lesions involving the conjunctiva and cornea, ranging from benign lesions to life-threatening malignancies. The authors reports recent evidence that topical chemotherapy has been showing complete tumor resolution and a low recurrence rate with less injury compared to surgical removal (15). Alessandro Comandone and coauthors present a phase II monoinstitutional study, which was aimed at assessing the effect of metronomic cyclophosphamide administration as second and further line of therapy in patients with metastatic soft tissue sarcomas. The study showed a stabilization of the disease in most cases and a good profile of toxicity. The authors discuss specific issues that deserve further investigation as well the specific classes of patients who might most benefit this approach (16). Finally, we asked Massimo Di Maio, Professor of Medical Oncology and Secretary of the Italian Association of Medical Oncology, AIOM, to comment on the value of health-related quality of life (QoL) and patient-reported outcomes (PROs) in oncology clinical trials (17). The article, written in a question \& answer, interview format, will provide readers with a timely overview on the crucial need to implement clinical trials with both QoL assessment and PROs along with methodological steps that will help us to inch closer to a more patient-centered approach. 


\section{REFERENCES}

1. Giordano A, Pinto C, Pentimalli F. Cancer research and care in the future post-COVID-19 era. Ann Oncol Res [Internet] 2021;1(1). Available from: https://www.annals-research-oncology.com/covid-19-still-on-center-stage-in-oncology-debates/.

2. Hans Henri P. Kluge. Statement - Catastrophic impact of COVID-19 on cancer care, World Health Organization, Regional Office for Europe [Internet] 2021. Available from: https:// www.euro.who.int/en/media-centre/sections/ statements/2021/statement-catastrophic-impact-of-covid-19-on-cancer-care.

3. The Lancet Oncology. COVID-19 and cancer: 1 year on. Lancet Oncol [Internet] 2021 Apr 1;22(4):411. Available from: https://www. thelancet.com/journals/lanonc/article/PIIS14702045(21)00148-0/fulltext.

4. Yusuf A, Sarfati D, Booth CM, et al. Cancer and COVID-19 vaccines: a complex global picture. Lancet Oncol [Internet] 2021 Apr;0(0). Available from: https://doi.org/10.1016/S1470-2045.

5. Desai A, Gainor JF, Hegde A, et al. COVID-19 vaccine guidance for patients with cancer participating in oncology clinical trials [Internet]. Vol. 18, Nature reviews. Clinical oncology. NLM (Medline) 2021. p. 313-9. Available from: https://pubmed.ncbi.nlm.nih.gov/33723371/.

6. Mangone L, GiorgiRossi P, Bisceglia I, Grilli R, Pinto C. The impact of COVID-19 on new cancer diagnoses. Ann Oncol Res 2021;(1).

7. Cagnazzo C, Testoni S, Cenna R, et al. Clinical research during COVID-19 spread: managing an emergency within a pandemic outbreak. Ann Oncol Res 2021;1.

8. Lasagna A, Secondino S, Agustoni F, et al. Cancer care at the time of COVID-19: practical approach to the management of patients during the early time of the pandemic in a large Italian community hospital. Ann Res Oncol 2021;1.

9. Fratino L, Serraino D. SARS-CoV-2 and Oncology. Ann Res Oncol 2021;1(2).

10. Cavanna L, Casali S, Citterio C, Gianlupi G, Pilla L. COVID-19 outbreak in Italy. report of 20 cancer patients with COVID-19 treated at home during the first wave. Ann Res Oncol 2021;1(2).

11. Meet an Italian Doctor Treating Coronavirus Patients at Home | TIME. Available from: https://time.com/5816874/italy-coronavirus-patients-treating-home/.

12. Fanni D, Demontis R, Saba L, et al. Pulmonary capillary hemangiomatosis induced by SARSCoV-2 infection. A case report. Ann Res Oncol 2021;1(2).

13. Perrone AM, Tesei M, De Crescenzo E, et al. Prognostic role of the primary treatment in the natural history of ovarian cancer: a pilot study. Ann Res Oncol 2021;1(2).

14. Mangone L, Bisceglia I, Sacchettini C, et al. Neuroendocrine neoplasms in Italy. Ann Res Oncol 2021;1(2).

15. Mallone F, Chicca A V., Sagnelli P, Sacchetti M, Lambiase A. New insights on the diagnosis and management of malignant tumors of the ocular surface. Ann Res Oncol 2021;1(2).

16. Comandone A, Boglione A, Comandone T, Giubellino E, Oliva. C, Bergnolo P. Metronomic continuous oral cyclophosphamide (CPM) as second and further line in metastatic soft tissue sarcomas (STS) of the adult. Ann Res Oncol 2021;1(2).

17. Di Maio M. Implementing clinical trials in Oncology with quality of life assessment and patient reported outcomes. An interview to explore the road towards a more patient-centered approach. Ann Res Oncol 2021;1(2). 